\title{
Herramienta e-learning para la programación de robots mediante entorno web
}

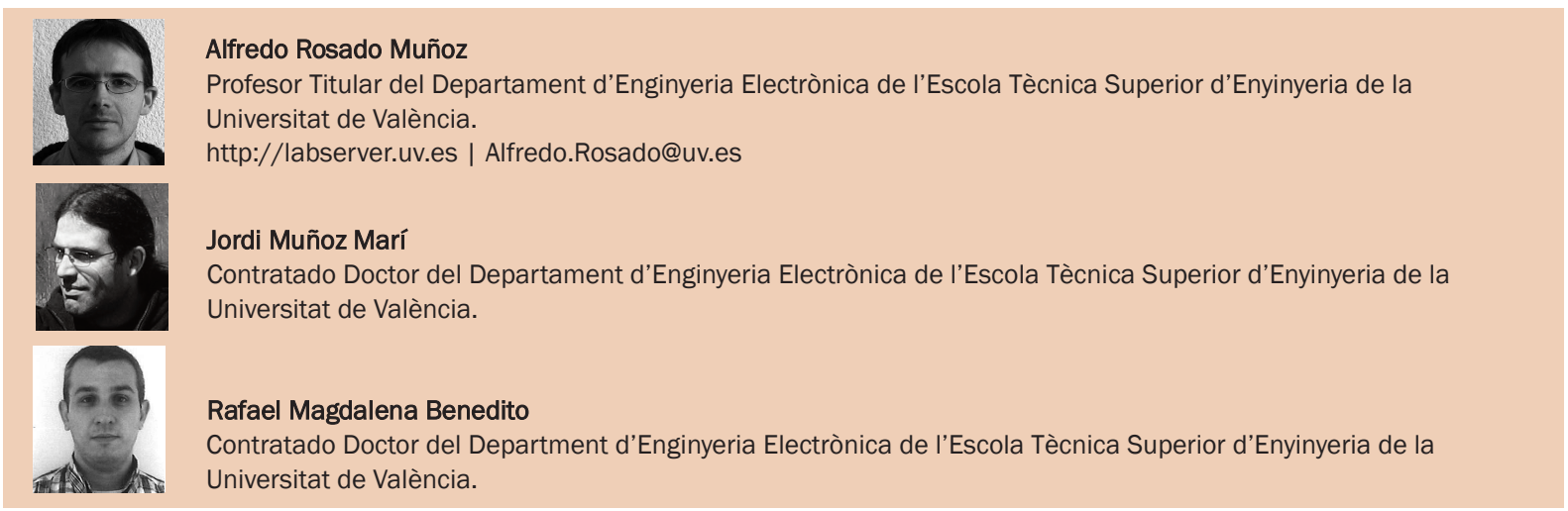

|Fecha presentación: 20/07/2007 | Aceptación: 03/10/2008 |Publicación: 22/12/2008

\begin{abstract}
Resumen
Este artículo describe los trabajos realizados para permitir a los estudiantes de diversas titulaciones de ingeniería la realización de prácticas a distancia en un laboratorio remoto desde un navegador web. Este hecho posibilita utilizar aparatos y maquinaria a distancia (inicialmente un robot industrial), pudiendo programarlos y visualizar el resultado de la programación realizada en tiempo real así como interactuar con la máquina. Adicionalmente, se propone al estudiante la realización de un curso de programación mediante diversas prácticas que debe realizar y el sistema web autoevalúa. Este trabajo ha requerido la integración de diversos elementos hardware y software.
\end{abstract}

Palabras clave: laboratorio remoto, programación de robots, e-learning, innovación educativa, autoevaluación

\section{Resum}

Aquest article descriu els treballs realitzats per a permetre als estudiants de diverses titulacions d'enginyeria la realització de pràctiques a distància a un laboratori remot des d'un navegador web. Aquest fet possibilita utilitzar aparells i maquinària a distància (inicialment un robot industrial), podent programar-los i visualitzar el resultat de la programació realitzada en temps real així com interactuar amb la màquina. Addicionalment, es proposa a l'estudiant la realització d'un curs de programació mitjançant diverses pràctiques que ha de realitzar i el sistema web autoevalúa. Aquest treball ha requerit la integració de diversos elements hardware i software.

Paraules clau: laboratori remot, programació de robots, e-learning, innovació educativa, autoavaluació

\begin{abstract}
This paper describes the work carried out to enable students of various engineering degrees the accomplishment of remote practices in a remote laboratory using a web browser. This fact allows the remote use of equipment and tools (in first stage an industrial robot), being able to program them and to visualize the output of the developed programs in real time as well as to interact with the machine. Additionally, it is offered to the student the accomplishment of a programming course by means of diverse practices to be performed and the
\end{abstract}


Web system provides information about the correctness of the developed lab session. This work has required the integration of different hardware and software elements.

Keywords: remote lab, robot programming, e-learning, educative innovation, self-evaluation

\section{Introducción}

El uso de robots está en aumento en los últimos años. La robótica industrial, si bien tiene su origen en la robótica clásica académica, ha evolucionado por sí misma hasta ser una rama de la ingeniería de valiosa importancia. Así, conjuntamente con la instalación de sistemas de automatización basados en PLC, cada vez es más frecuente encontrar robots para la realización de tareas mecánicas repetitivas o peligrosas para las personas, tales como la paletización, la soldadura en empresas del sector metalúrgico, el manejo de cajas de fruta en centrales hortofrutícolas, etc. Por todo ello, resulta patente la necesidad de introducir a los estudiantes de ingeniería en el entorno de la robótica industrial, para mostrarles sus capacidades, sus particularidades, y sus métodos de programación.

El principal objetivo de este proyecto consiste en poder facilitar el acceso de los estudiantes a los recursos necesarios para poder realizar la programación de robots. Un robot industrial posee un coste elevado, lo que implica que los centros docentes no siempre puedan disponer de instalaciones adecuadas para su manejo en aulas. Por lo tanto, este trabajo propone la realización de una plataforma de aprendizaje mediante la cual los alumnos puedan aprender a programar utilizando un robot real. A través de la plataforma de aprendizaje propuesta se persiguen varios objetivos; en primer lugar, dotar al robot de elementos de accionamiento y sensado que permitan ofrecer una serie de prácticas muy similares a los trabajos que realizan los robots en la industria; por otro lado, realizar una interfaz de acceso al robot de modo que sea posible la programación del mismo, tanto de forma remota para realización de prácticas no presenciales como desde la misma aula donde se encuentra el robot (prácticas presenciales). El hecho de ofrecer prácticas no presenciales resulta muy importante, ya que se pretende que el estudiante pueda acceder al robot a cualquier hora del día y desde cualquier lugar que disponga de conexión a Internet. De este modo, es posible hacer que un único recurso hardware de elevado coste pueda ser empleado por un gran número de usuarios, lo que hace que la inversión realizada en el equipamiento sea más rentable en términos de aprovechamiento por parte de los alumnos.

Adicionalmente, el entorno web de realización de prácticas remotas pretende ser una plataforma de auto aprendizaje, por lo que se desea ofrecer al estudiante el seguimiento de un determinado número de prácticas propuestas, y que éstas sean autoevaluadas desde la plataforma web, indicando al estudiante si ha realizado la práctica correctamente, y en caso contrario, dónde se puede haber equivocado. Para ello, será necesario crear un entorno web amigable mediante el cual sea posible programar y utilizar el robot, realizar una reserva previa del equipo (dado que se va a utilizar un equipo real, no es posible que dos usuarios estén conectados al mismo tiempo), gestionar el acceso de usuarios y obtener datos sobre el uso del sistema.

De forma concreta, las características esenciales del sistema propuesto son:

1. Diseño de un sistema mediante el cual se puedan realizar prácticas de programación de robots mediante el acceso ordenado al robot desde diferentes ubicaciones, tanto locales (mismo laboratorio con varios PC) como remotas (cualquier PC con acceso a Internet).

2. Maximizar la utilización del robot en el tiempo.

3. Robustez. El sistema debe estar preparado para responder ante posibles malos usos de los usuarios. Dado que se trata de un elemento móvil, podría darse el caso que los movimientos indicados por un usuario dañaran la instalación, cosa que debe estar prevista para evitar que ocurra.

4. Económico. Una vez se ha realizado la instalación del sistema, su mantenimiento no debe suponer un alto coste.

5. Seguridad. El sistema debe detenerse en caso de que alguna persona intente acceder al recinto donde se encuentra el robot en movimiento.

6. El sistema de acceso mediante web debe proporcionar la mayor información posible para facilitar el aprendizaje del usuario.

Existen pocas experiencias en el campo de la programación de robots para lograr controlarlos a través de Internet. En el caso de Candelas et al. (2006) permiten el acceso a un simulador virtual del robot, pero no al robot físico. Por otro lado (Šafaric et al. 2005) propusieron un sistema similar al que se expone aquí, pero para un robot no industrializado y mediante una interfaz poco estandarizada a través de múltiples conexiones, lo que lo hacía poco flexible, además de no disponer de prácticas de laboratorio estandarizadas y autoevaluables.

En cambio, de forma más general, el planteamiento de poder acceder a recursos remotos a través de Internet con fines educativos ya viene siendo un campo de trabajo desde hace varios años. Existen diversas publicaciones interesantes donde se reflejan propuestas similares a la de este trabajo: Fjeldly y Shur (2003) describen numerosas posibilidades para poder realizar experimentos de electrónica a través de Internet; Gustavsson (2002) obtuvo el premio al mejor trabajo por el acceso remoto al control de experimentos de electricidad por su novedad e interés; Hercog et al. (2005) y Herrera et al. (2006) también realizan propuestas encaminadas en este sentido. Esta gran actividad en este tipo de trabajos muestra el enorme interés que suscita y las posibilidades que se pueden plantear con el acceso remoto a elementos hardware.

\section{Metodología}

Fruto de ese análisis, se ha diseñado una plataforma web bajo las premisas de simplicidad y fácil comprensión. Para poder disponer de un entorno web potente y con posibilidades de gestión de usuarios, almacenamiento de datos, registros de conexión, etc., ha sido necesaria la instalación de un servidor web bajo PC operado en sistema operativo Linux, lo que proporciona de este modo máxima robustez y seguridad, además de que se trata de software libre. La web ha sido realizada en lenguaje PHP y la gestión de base de datos en MySQL. Además, para el control hardware del sistema se emplea la tarjeta CP343-1 IT de Siemens, que proporciona la comunicación directa con valores de memoria interna y de entradas y salidas del PLC, que a su vez controla el robot y los diversos elementos hardware del sistema. El sistema incor- 


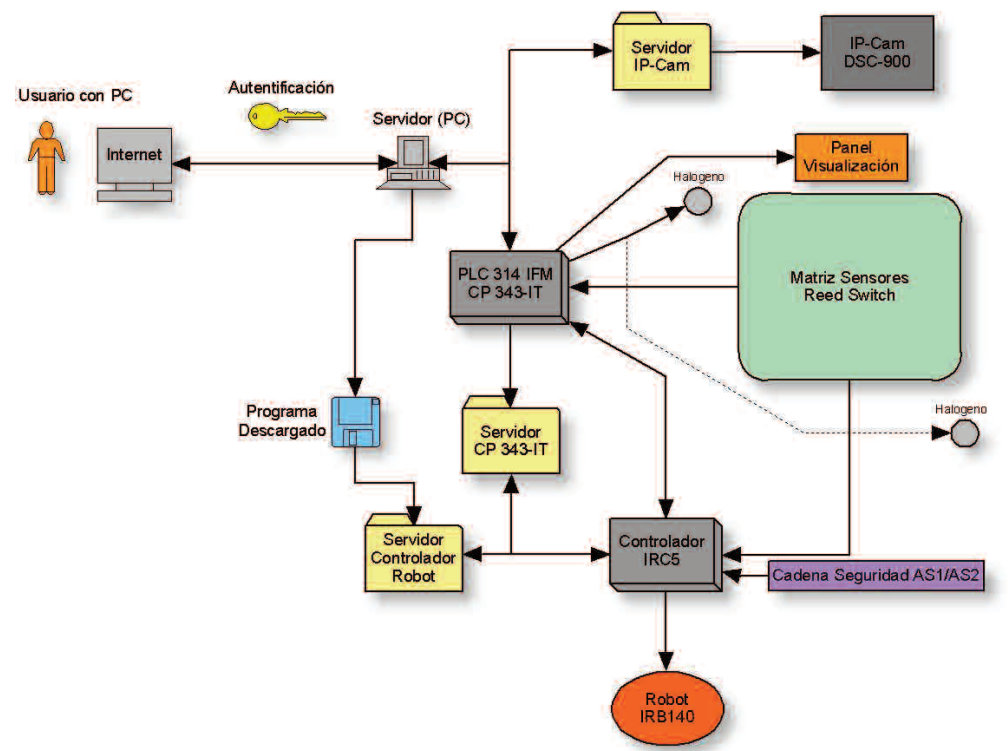

Figura 1. Estructura de la plataforma de aprendizaje para el acceso a laboratorios de forma remota

pora también una cámara IP para visualización en tiempo real y un sistema de iluminación propio, que se activa cada vez que accede un usuario. La Figura 1 muestra el esquema básico de la estructura general del sistema planteado.

Con el entorno web propuesto, un usuario de la plataforma web debería realizar los siguientes pasos:

- Conocer las prácticas que debe realizar, descargar el guión de prácticas y desarrollar el programa de la práctica correspondiente para el robot en lenguaje RAPID. Todo ello se realizaría previamente a la conexión al servidor web, ya que son tareas a realizar de modo off-line.

- Autentificación para acceder a la plataforma web. Inicialmente mediante una gestión de usuarios propia, pero con la previsión de enlazar con el acceso a través de la plataforma Aula Virtual de la Universitat de València.

- Seleccionar la práctica que se desea realizar.

- Se accede a una nueva pantalla donde se realiza la descarga al robot del fichero de programación RAPID que el estudiante ha elaborado.

- Una vez se ha cargado el fichero en el controlador del robot, se accede al servidor web del PLC, el cual ofrece una pantalla de control del robot con la posibilidad de interaccionar con él mediante tres botones:

- Inicio: Para comenzar a ejecutar el programa cargado

- Paro: Para detener en cualquier momento el programa

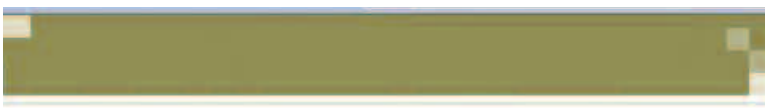

Guia de uso Nuevo usuario Reservar sesión Inicio

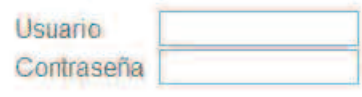

Enviar

Figura 2. 1. Pantalla de entrada al sistema web
- Nueva Práctica: Si se desea realizar otra práctica o volver a descargar el programa con las modificaciones oportunas, en caso de detectar errores

- En la parte superior izquierda de la pantalla descrita en el punto anterior se muestra la imagen web del laboratorio proporcionada por la cámara IP (Figura 2.2). En la parte superior derecha se visualiza el marco donde se encuentran los 3 botones de interacción entre el usuario y el robot (inicio, paro y nueva práctica), así como diversa información que cambia dinámicamente según las acciones que realiza el robot, tales como:

- Estado del robot: activo/inactivo

- Estado del programa: Programa cargado/descargado

- Estado de la cabeza: Electroimán activo/inactivo

- Errores: Informa si se detectan errores durante la ejecución del programa

- Programa correcto: Si la práctica propuesta se ha realizado con éxito

- Programa incorrecto. Ha habido errores en el programa y no se han realizado los movimientos de la forma que se requería en el guión de la práctica

Por último, en la parte inferior de la pantalla se muestran las señales necesarias para que el usuario conozca cómo se está desarrollando la práctica. Por ejemplo, para la práctica de atornillado propuesta (práctica 3 , donde se propone
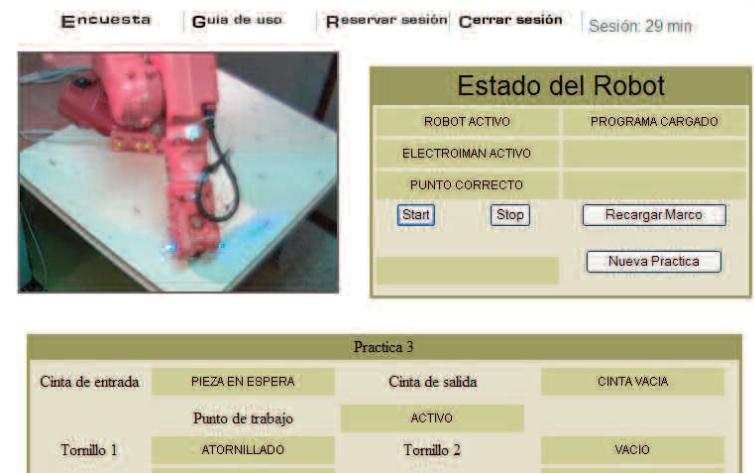

Figura 2.2. Pantalla de trabajo durante la realización de una práctica 


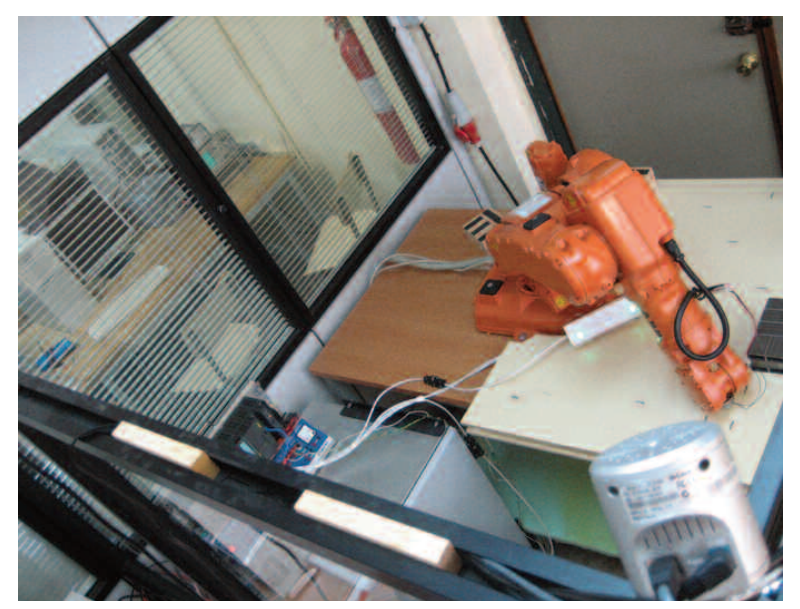

Figura 3. Fotografía del laboratorio de control de robots accesible desde web

un símil a este proceso industrial), se informa del estado de la cinta transportadora de entrada de pieza, estado del puesto de trabajo, el atornillado de la pieza y su posicionamiento final en la cinta de salida. Esta verificación se realiza a través del PLC, encargado de revisar que para cada práctica propuesta se han alcanzado correctamente las posiciones indicadas en el guión de prácticas, ofreciendo una autoevaluación inmediata sobre las actividades realizadas. Las Figuras 2.1. y 2.2 muestran varias imágenes del entorno web propuesto y la Figura 3 una fotografía de la disposición real del robot.

El sistema está accesible para todos aquellos que lo deseen en la dirección web: http://labserver.uv.es. En esta dirección encontrarán las instrucciones necesarias para poder introducirse al sistema como usuario invitado y así poder observar la potencialidad de la plataforma desarrollada.

\section{Conclusiones}

Con el desarrollo de este proyecto se pretende ofrecer un servicio novedoso a los estudiantes, permitiendo además que se facilite el acceso a recursos de elevado coste, donde resulta complejo su uso presencial. Esta metodología pretende, por otro lado, iniciar una aproximación a la docencia acorde con el Espacio Europeo de Educación Superior (EEES), para que una vez implantados los nuevos títulos de Grado y Máster, se pueda planificar la docencia con la ventaja de haber realizado experiencias docentes previas.

El alumno que lo desee pueda reservar el robot durante un periodo de 30 minutos, descargar y ejecutar el programa, teniendo la posibilidad de visualizarlo a través de cámara IP. Por otro lado, el servidor web se ha programado para ofrecer estadísticas de uso.

Cabe reseñar que, en este proyecto, se ha buscado constantemente la flexibilidad y el diseño de un sistema que sea fácilmente adaptable a cualquier tipo de experimento donde se precise el empleo de hardware difícilmente accesible para los estudiantes, con lo que esperamos poder implantar esta metodología en otras plataformas hardware.

\section{Bibliografía}

Candelas Herías, Francisco Andrés.; Jara Bravo, Carlos Alberto; Torres Medina, Fernando (2006). Flexible virtual and remote laboratory for teaching Robotics. En A. Méndez-Vilas; A. Solano Martín; J.A. Mesa González (Ed.). Current Developments in Technology-Assisted Education. Badajoz: Formatex, pp. 1.959-1.963.

Damas, M.; Pomares, H.; Tarifa, J.A.; Jiménez Mesa, G.; y Roldán Herencia, M.V. (2006). Virtual laboratory for the supervision and control of scale models of industrial processes. En A. Méndez-Vilas; A. Solano Martín; J.A. Mesa González (Ed.). Current Developments in Technology-Assisted Education. Badajoz: Formatex, pp. 1.342-1.346.

Fjeldly,Tor A.; Shur, Michael S. (2003). Lab on the Web: Running Real Electronics Experiments Via the Internet. New York: Wiley - IEEES Press.

Gustavsson, Ingvar (2002). A Remote Laboratory for Electrical Experiments. Proceedings of the 2002 American Society for Engineering Education Annual Conference \& Exposition. American Society for Engineering Education. http://www.bth.se/fou/forskinfo.nsf/alfs/c1ef95obb4 8 e1272c1256c710038cc2c/\$file/2002-C-ASEEIGU.pdf

Hercog, Darko; Gergic, Bojan; Matko, Vojko (2005). Remote Lab for Electric Drives. IEEE International Symposium on Industrial Electronics, june 20-23, pp. 1.685-1.690. http://ieeexplore.ieee.org/stamp/stamp.jsp?arnumbe$\mathrm{r}=01529185$

Herrera, Oriel A.; Alves, Gustavo R.; Fuller, David; Aldunate, Roberto.G. (2006). Remote Lab Experiments: Opening Possibilities for Distance Learning in Engineering Fields. En Deepak Kumer y Joe Turner (Ed.). Education for the 21st Century - Impact of ICT and Digital Resources. IFIP 19th World Computer Congress, TC-3, Education, August 21-24, 2006, Santiago, Chile (Hardcover). Springer Boston, pp. 321-325.

Šafaric, Riko.; Truntic, Mitja.; Hercog, Dark.; Pacnik, Gregor (2005). Control and robotics remote laboratory for engineering education. International Journal on Online Engineering (iJOE). Vol.1, 1. http://www.i-joe.org

| Cita recomendada de este artículo

Rosado Muñoz, Alfredo.; Muñoz-Marí, Jordi y Magdalena Benito, Rafael (2008). Herramienta e-learning para la programación de robots mediante entorno web. @tic. revista d'innovació educativa ( $\left.n^{\circ} 1\right)$ http://ojs.uv.es/index.php/attic/article/view/48/45 Fecha de consulta, $\mathrm{dd} / \mathrm{mm} / \mathrm{aa}$ 